\title{
CHALLENGES AND ISSUES OF EYE DISEASES IN HUMAN
}

Dr. S. Veena

Professor,

Department of CSE

S.A. Engineering College, Thiruverkadu, Chennai, Tamil Nadu, India
R.Krupali

UG Student, Department of CSE

S.A. Engineering College, Thiruverkadu, Chennai, Tamil Nadu, India.

ABSTRACT- Eye diseases are common among people of all ages ranging from children to old. Eye diseases can be defined as an impairment or abnormal functioning of the eye which leads to visual disturbance. Most eye diseases cause blurred vision and loss of eyesight. This paper presents a survey on challenges and issues of eye diseases Like cataract, glaucoma, diabetic retinopathy, retinoblastoma, age related macular degeneration. And this paper also discusses the technology,pros and cons of existing systems.

Keywords - Raspberry Pi NoIRInfrared, Camera, retinal image, RaspberryPi2, Eye Disease, cataracts, glaucoma, retinal disorders

\section{INTRODUCTION}

Eye diseases are the common disease people are facing now a day. Even people of all ages ranging from children to old are affected due to eye disease. Some diseases become very cautious if they are untreated at an early stage. Some eye problems are minor and don't last long but some can lead to a permanent loss of vision.To overcome such problems early detection and treatment could prevent vision loss.Eye problems affect almost everyone at some point in their lives. For many, eye disorders have become almost a part of their daily lives. Early detection and treatment of retinal eye diseases is critical to preventing visual loss. Traditionally, retinal disease detection techniques were based on manual description. The World Health Organization or WHO finds that there were millions of visually impaired people all over the world. The number of cases of blindness has been extensively reduced in recent years, it is estimated that $82 \%$ of the cases of visual impairment are preventable or treatable. Retina is affected by several disorders which may be vision changes with aging or diabetics. Many changes are common and can often be corrected at an early stage and can be easily identified by humans.

Prevention of blindness is an important challenge around the world. Cataracts, glaucoma and age-related macular degeneration are the three major diseases that cause blindness. Vision 2020 pointed out that there are about 285 million visually impaired people worldwide. Therefore, early detection is particularly important when improving eye care services.

D.Mahalakshmi

UG Student,

Department of CSE

S.A. Engineering College,

Thiruverkadu, Chennai,

Tamil Nadu, India.

\author{
B.Preetha \\ UG Student, \\ Department of CSE \\ S.A.EngineeringCollege, \\ Thiruverkadu, Chennai, \\ Tamil Nadu, India.
}

This paper encounters different eye diseases and detection methodologies for efficient and timely manner diagnosis. Retinal images are widely used by ophthalmologists and primary care physicians for screening eye diseases, as they are not direct and harmless. For that fundus images of the eye are used mostly.This does not cause irritation in eyes and feel comfortable for humans to use it effectively.

\section{RETINAL DISEASES AND PREVENTION}

\section{CATARACT}

Cataracts are clouding of the lens in your eye and changes the original Colour appearance of pupil. They can develop in one or both eyes. The lens is usually clear and the color of the eye sits behind the iris. The lens helps focus the light to create a sharp image in the retina behind the eye and changes the shape to allow you to see close-ups. A cataract acts like a frosted glass coating that scatters light, causing blurring and lack of clarity. If it continues the loss of eyesight appears and leads to complete blindness this may cause due to medical conditions like diabetics or any eye surgery done earlier.

\section{SYMPTOMS OF CATARACT}

- Blurring

- Dazzled by lights

- Double vision

\section{PREVENTION}

Proper and prior treatment should be taken to overcome cataract. As if it is affected by diabetics it becomes worse if they are untreated. Early detection can be made to overcome this disease and consumption of prescribed tablets.

\section{GLAUCOMA}

Glaucoma is the name given to the group of eye conditions that damage the optic nerve at the point of departure of the eye. The optic nerve carries images from the retina, the light sensitive membrane attached to the inner surface of the eye, to the brain. In this there are conditions that can be cured if it is in early stage of detection. 


\section{SYMPTOMS OF CATARACT}

- Loss of peripheral vision

- Seems to be normal

- Loss of central vision

\section{PREVENTION}

Regular eye test should be taken and early detection of disease should be taken to prevent blindness. There is no cure for this disease so you want to do all you can early to prevent the irreversible effects.

\section{DIABETIC RETINOPATHY}

Diabetes can lead to a condition called diabetic retinopathy.This disease is caused due to damage in the blood vessels which is caused at the back of the eye in retina. which can eventually lead to blindness. It is an ocular manifestation of systemic disease, which affects up to $80 \%$ of all patients who have had diabetes for 10 years or more.

\section{SYMPTOMS}

- Floaters and spots in your vision

- Blurred or cloudy vision

- Dark or empty spot in the center of the vision

- Difficulty seeing at night

\section{PREVENTION}

It can beprevented if proper treatment and monitoring of the eyes is carried out on a regular basis. Sugar and blood pressure level should be maintained. Early detection of disease should be taken and regular exercise.

\section{RETINOBLASTOMA}

Retinoblastoma is a rare eye cancer disease which generally affects children under 5 years old. Retinoblastoma is the most common type of eye cancer in children. It is treatable, but it should be detected earlier.The eyes have cells called retinoblasts that divide into new cells and fill the retina. Eventually, these cells cease the dividing process and turn into mature retinal cells. Sometimes, something goes wrong with this process and rather than maturing into special cells that detect light, some of the retinoblasts continue to divide and grow out of control, forming a cancer known as retinoblastoma.

\section{SYMPTOMS}

- A White reflex

- A squint

- Red, sore or swollen eye without infection

- A change in colour of the iris

\section{PREVENTION}

Retinoblastomas are detected and treated before they have a chance to spread outside the eyeball, but retinoblastoma cells can occasionally spread to other parts of the body spreading the cancer. Prior precaution should be taken and can be cured at early stage.

\section{AGE RELATED MACULAR DEGENERATION(ARMD)}

Age-related macular degeneration or AMD is an eye disorder and a most important source of vision loss among people age 50 and older and it causes pain to the macula which is a small spot near the center of the retina and the part of the eye desired for sharp, central vision. AMD affects very slowly so that vision loss does not happen for long. Sometimes the disease progresses rapidly and can lead to vision loss in one or both eyes.

\section{SYMPTOMS}

- Blurriness

- Dark areas

- Distorted vision

- Causes due to smoking

\section{PREVENTION}

While there is no cure for macular degeneration at this time, there are steps that can be taken to reduce the risk or slow the progression of the disease such Make changes to your diet, exercise regularly, quit smoking and protect your eyes from UV light. Regular eye check up should be taken to overcome this loss of vision.

\section{EXISTING WORK AND APPROACHES}

Some of the exiting survey works on eye disease are discussed below

M. Usman Akram, Sundas Mujtaba and Anam Tariq [1] proposed the automatic drusen segmentation in fundus films for the detection of age-related macular degeneration. Agerelated macular degeneration (ARMD) is an eye disease caused by the presence of drusen in the retina, and may cause blindness. Early detection of ARMD using a computerized system can restore patient vision. Ophthalmologists can achieve this method for screening ARMD. This article presents a new method for accurately detecting drusen in colored retina images. The system generates a retina image to extract all the filter bank and eliminates invalid pixels that appear to be due to Drewson's resemblance to the optical disk. The system refers to each region with many features, and then uses support vector machines to classify these regions as drusen and non-drusen. Performance is evaluated by testing in the STARE database using parameters such as performance and accuracy.

Mimi Yang, Ji-Jiang Yang, Qianyang Zhang, Yu Nguyangian Li [2] proposed to classify the retina image for automatic cataract detection. This paper proposes to use a neural network classifier for automated cataract detection based on the classification of retinal images. This method is pre-processed, feature-built. Extraction and classification construction. In the pre-processing area, an advanced topdown cap modification is used to improve the contrast between the foreground and the object, and a tripartite filter is used to reduce noise in the image. The two-layer back propagation (PP) is characterized by a neural network. 
Cataracts can be distinguished as normal, mild, medium or severe.

R. Geetharamani, Lakshmi Balasubramanian [3] proposed a new computational approach for automated prognosis of diabetic patients using retinal image analysis and data mining techniques to classify retinal images into normal, diabetic retinopathy and glaucoma. Three feature matching and sixteen classification algorithms were analyzed and used to identify the contributing features that yield the best predictive results. Our results demonstrate that C4.5 and random tree classification techniques produce $100 \%$ maximum multi-class classification training accuracy in classifying 45 images from the gold standard database. Furthermore, the Fisher's ratio algorithm reveals the lowest and optimal predictive features in retinal image training data.

Muhammad Salman Haleem, Liangxiu Hana, Jano van Hemertb, Baihua Li [4] proposed the paper Automatic extraction of retinal features from color retinal images for diagnosis of glaucoma. Techniques used to diagnose glaucoma include angioscopy, intraocular pressure or IOP measurement using a tonometer, which are manually prepared by doctors. Complete diagnostic tests for glaucoma are usually followed by optic nerve head $(\mathrm{ONH})$ imaging tests, which require regular monitoring, which is expensive and time-efficient. The accuracy and consistency of the diagnosis is controlled by the field knowledge of ophthalmologists.

Weiming Fan, Ruifang Shen, Qinyan Zhang, Ji-Jiang Yang, Jianqiang Li [5] proposed cataract quality and classification based on primary instrument analysis. Based on the classical classification system of cataract quality and classification, they add a step (PCA) to the system and make it more accurate considering the computational cost and training time of a classifier. Although there is a slight decrease in the accuracy rate, the computational speed is greatly improved. As the number of images involved in the structure of a classifier increases, the importance of PCA transformation becomes more significant. Automated cataract detection classification system based on retinal fundus images: threestep fundus image pre-processing, feature extraction and classification.

Xinting Gao, Stephen Lin and Tien Yin Wong [6] proposed the paper Automated feature learning to standardize atomic cataracts based on deep learning. This paper proposes a new method for the standardization of nuclear cataracts based on automatic feature learning. It is difficult to find the right features, and this study develops a new approach that directly addresses this problem in a systematic and generic way, rather than seeking out heuristic features. In deep learning, discriminative features that describe high-level semantic information are effectively extracted. This approach has the potential to be used for other eye diseases as well; Different artifacts are used in the optic cup or disk section to evaluate the sequelae of glaucoma and to detect drusen to measure AMD. The features extracted through this type of deep learning approach can lead to improved performance in these cases.

Dharitri Deka, Jyoti Prakash Medhi, S. R. Nirmala [7] proposed the paper Detection of macula and fovea for disease analysis in color finance films. The macula and fovea play an important role in the diagnosis of various retinal diseases. Any irregular fall in the macular area affects the central vision and slowly leads to blindness. Here they presented a good method for finding macula and foa. PV The macula was translated using the framework property and was found to be the core of the fovea macula. They tested normal and diseased images using the DRIVE, MESSIDOR, DIARETDB1, HRF, and STARE databases.

D Huiying Liu, Yanwu Xu, Damon W.K. Wong, and Jiang Liu [8] proposed the paper Develop a cutting-based Truesan section for age-related macular degeneration detection. This article proposes a novel Truesan section method. It often occurs in the elderly and is the third largest disease leading to blindness after cataracts and glaucoma. The main characteristic of this method is the use of the cross cut to find the boundaries of the drusen. The initial seeds for the Grow Cut are automatically determined. This method first finds the local maximum and minimum points. Possible points are that they are potentially Druzen, and then they are classified as Drusen or non-Drusen.

Yoo Joo, Kyuoki, HuigiLi [9] proposed autonomic cataract classification using a deep neural network with distinct state change. Automated cataract detection and standardization methods are proposed in this paper. Without prior knowledge, the remaining neural networks with either DST (DST-RESNET) or EDST (EST-RESNET) are proposed. With or without prior knowledge, the proposed DST and EDST strategy can prevent excessive objects and reduce storage memory during network training and implementation, and neural networks with these techniques achieve sophisticated accuracy in cataract detection and grading. The test results indicate that the integrated results always achieve the best performance accuracy, and feature extraction classification methods based on previous knowledge are well suited for the complex medical image classification task. Using this technique, pre-diagnosis can be taken and vision loss can be prevented. 
International Journal of Engineering Applied Sciences and Technology, 2020

Vol. 4, Issue 11, ISSN No. 2455-2143, Pages 582-586

Published Online March 2020 in IJEAST (http://www.ijeast.com)

\begin{tabular}{|c|c|c|c|c|}
\hline S.NO & TITLE & $\begin{array}{l}\text { TECHNOLOGIES } \\
\text { TOOLS USED }\end{array}$ & ADVANTAGES & DISADVANTAGES \\
\hline 1. & $\begin{array}{l}\text { Automated drusen } \\
\text { segmentation in fundus images } \\
\text { for diagnosing age related } \\
\text { macular degeneration }\end{array}$ & Machine learning & Highly efficient. & High risk. \\
\hline 2. & $\begin{array}{l}\text { Classification of retinal image } \\
\text { for Automatic cataract } \\
\text { detection. }\end{array}$ & Deep learning & $\begin{array}{l}\text { It uses neural network } \\
\text { classifier }\end{array}$ & $\begin{array}{l}\text { Difficult to identify the } \\
\text { type of cataract }\end{array}$ \\
\hline 3. & $\begin{array}{l}\text { Automatic prediction of } \\
\text { diabetics retinopathy and } \\
\text { glaucoma using data mining. }\end{array}$ & $\begin{array}{l}\text { Data mining,image } \\
\text { processing }\end{array}$ & Accurate result & $\begin{array}{l}\text { It takes } 30 \text { minutes to } \\
\text { maximize the sensibility. }\end{array}$ \\
\hline 4. & $\begin{array}{l}\text { Automatic extraction of retinal } \\
\text { features from color retinal } \\
\text { images for Glaucoma } \\
\text { Diagnosis. }\end{array}$ & Image processing Iot & $\begin{array}{l}\text { The accuracy and } \\
\text { consistency of } \\
\text { diagnosis is restricted }\end{array}$ & $\begin{array}{l}\text { Expensive and time } \\
\text { inefficient }\end{array}$ \\
\hline 5. & $\begin{array}{l}\text { Principal Component Analysis } \\
\text { Based Cataract Grading and } \\
\text { Classification. }\end{array}$ & Image processing & $\begin{array}{l}\text { computation speed and } \\
\text { cost get improved }\end{array}$ & $\begin{array}{l}\text { slightly decrease in } \\
\text { accuracy ratio. }\end{array}$ \\
\hline 6. & $\begin{array}{l}\text { Automatic Feature Learning to } \\
\text { Grade Nuclear Cataracts Based } \\
\text { on Deep Learning, }\end{array}$ & Deep learning & improved performance & $\begin{array}{l}\text { difficult to find the right } \\
\text { features }\end{array}$ \\
\hline 7. & $\begin{array}{l}\text { Detection of Macula and Fovea } \\
\text { for Disease Analysis in Color } \\
\text { Fundus Images. }\end{array}$ & $\begin{array}{l}\text { DRIVE, MESSIDOR, } \\
\text { DIARETDB1, HRF, } \\
\text { STARE databases }\end{array}$ & $\begin{array}{l}\text { Good method of } \\
\text { detection of macula } \\
\text { fovea disease. }\end{array}$ & $\begin{array}{l}\text { Tested in both normal } \\
\text { and diseased images. }\end{array}$ \\
\hline 8. & $\begin{array}{l}\text { Grow cut-based Drusen } \\
\text { Segmentation for Age-related } \\
\text { Macular Degeneration } \\
\text { Detection }\end{array}$ & Image processing & $\begin{array}{l}\text { effectiveness of the } \\
\text { method. }\end{array}$ & $\begin{array}{l}\text { tested on a manually } \\
\text { labeled dataset. }\end{array}$ \\
\hline 9. & $\begin{array}{l}\text { Automatic Cataract } \\
\text { Classification Using Deep } \\
\text { Neural Network With Discrete } \\
\text { State Transition }\end{array}$ & Image processing & $\begin{array}{l}\text { achieve state-of-the-art } \\
\text { accuracy in cataract } \\
\text { detection. }\end{array}$ & $\begin{array}{l}\text { reduce storage memory } \\
\text { during network training. }\end{array}$ \\
\hline
\end{tabular}

\section{CONCLUSION}

There are many retinal diseases in this world, which causes harm to our human eye and leads to loss of vision. This survey paper proposes studyon different eye disease and methods used for detection of retinal diseases. Finally a comparison table is presented with all the existing methodologies that are tabulated and mentioned along with their advantages and drawbacks. To detect different types of eye diseases that can be implemented using IOT technology using NIOR camera. Here we conclude that 
the different types of eye disease can be detected and treated at early stage and can be prevent the loss of vision.

\section{ACKNOWLEDGMENT}

We sincerely thank the management of S.A Engineering college, advisor, principal and faculty members for guiding us in doing our research and motivating us to do more such researches in future.

\section{REFERENCE}

[1] Akram Usman.M,Mujtaba Sundus,Tariq Anam(2014)," Automated drusen segmentation in fundus images for diagnosing age related macular degeneration,IEEE Publishing International Conference on electronics,computer andcomputation", 23

jan 2013,DOI:10.1109/ICECCO.2013.6718217.

[2] Yang Meimei,YANG Ji-Jiang,Zhang Qinyan, Niu Yu,Li Jianqiang(2014)," Classification of retinal image for Automatic cataract detection",IEEE Publishing 15 International Conference on e-Health Networking,Applications and Services, 27 jan 2014,DOI:10.1109/HealthCom.2013.6720761

[3] Ramani Geetha.R,Balasubramanian Lakshmi,Shomona Gracia Jacob(2013)," Automatic prediction of diabetics retinopathy and glaucoma using data mining",IEEE Publishing International Conference on Machine Vision and Image Processing 1 10ng, 4 feb 2013,DOI:10.1109/MVIP.2012.6428782.

[4] Haleem MS,Han L,van Hemert J,Li $\mathrm{B}(2013)$ "Automatic extraction of retinal features from color retinal images for Glaucoma Diagnosis"oct-dec

2013,DOI:10.1016/j.compmedimag.2013.09.005.Ep ub 2013 sep 27

[5] Fan Weiming,Shen Ruifang,Zhang Qinyan,Yng JiJiang,Li Jianqiang(2016)“Principal Component Analysis Based Cataract Grading and Classification "IEEE International Conference on E-Health Networking,Applications and Services, 19 april 2016,DOI:10.1109/HealthCom.2015.7454545

[6] Gao Xinting,Lin Stephen and Tien Yin Wong(2015),“Automatic Feature Learning to Grade Nuclear Cataracts Based on Deep Learning",IEEE publishing transaction on Biomedical
Engineering, 11

june

2015, Volume: 62,Issue:11,DOI:10.1109/TBME.2015.2444389.

[7] Deka Dharitri, Jyoti Prakash Medhi, Nirmala S.R(2015)"Detection of Macula and Fovea for Disease Analysis in Color Fundus Images",IEEE Publishing $2^{\text {nd }}$ International Conference on recent Trends in Information System,3 sep 2015,DOI:10.1109/ReTIS.2015.7232883

[8] Liu Huiying,Xu Yanwu,W.K.Wong, and Jiang Liu(2015), “Growcut-based Drusen Segmentation for Age-related Macular Degeneration Detection"IEEE Publishing Visual Communication and Image Processing Conference, $2^{\text {nd }}$ march 2015,DOI:101109/VCIP.2014.7051529

[9] Zhou Yue,Li Guoqi,Hu,Li Huiqi(2020),"Automatic Cataract Classification Using Deep Neural Network With Discrete State Transition",IEEE Transaction on Medical Imaging,feb 2020,Volume:39,Issue:2,DOI:10.1109/TMI.2019.29 28229

[10] Annette K.Hoskin,Stephanio L.Watson,Lisa Keay(2019),"Eye registries-A Systematic Review ",nov 2019, Volume:50,Issue: 11,DOI:10.1016/j.injuiry.2019.07.019

[11] Kuti Asiedu,Selassie K.Dzematu,Samuel Kyel(2019),"Clinical Subtypes of dry eye in youthful Clinical sample in Ghana",April 2019,Volume:42,Issue:2,DOI:10.1016/j.clae.2018.1 0.005

[12] Mehrdad Mohanmadpour,Amirreza Shaabani,Reza Mirshahi(2019),"Updates on management of pediatric cataract",jun 2019,Volume:31,Issue:2,DOI:10.1016/J.J010.2018. 11.005

[13] Alamelu M,Balaji R,Mithun j,Hariharan M(2019),"DR Vision Analysis using image identification using service analysis approach”,2019,Volume:165,DOI:10.1016/j.trocs.2 020.01 .008

[14] Kikut Ava,Vaughn Marquis,Jan o'Brien(2020),"Evaluation of a multimedia marketing campaign to engage African American patients in glaucoma screening",march 2020,Volume:17,Article id:101057,DOI:10.1016/j.pmedr.2020.101057

[15] Cherry Ahmed,Aridi Mona,Ghach Wiffam(2019),"Prevalent and risk factors of symptomatic dry eye disease in Lebanon", $9^{\text {th }}$ aug 2019,DOI:10.1016/j.clae.2019.08.0. 